\title{
(6) OPEN ACCESS \\ Revisiting the vicious circle of dry eye disease: a focus on the pathophysiology of meibomian gland dysfunction
}

\author{
Christophe Baudouin, ${ }^{1,2}$ Elisabeth M Messmer, ${ }^{3}$ Pasquale Aragona, ${ }^{4}$ Gerd Geerling, ${ }_{1}^{5}$ \\ Yonca A Akova, ${ }^{6}$ José Benítez-del-Castillo, ${ }^{7}$ Kostas G Boboridis, ${ }^{8}$ \\ Jesús Merayo-Lloves, ${ }^{9}$ Maurizio Rolando, ${ }^{10}$ Marc Labetoulle ${ }^{11}$
}

For numbered affiliations see end of article.

\section{Correspondence to}

Professor Christophe Baudouin, Quinze-Vingts National Ophthalmology Hospital, 28 rue de Charenton, Paris 75012, France; cbaudouin@15-20.fr

Received 30 June 2015 Revised 29 October 2015 Accepted 28 November 2015 Published Online First 18 January 2016

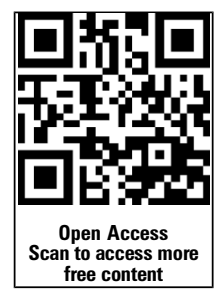

CrossMark

To cite: Baudouin $C$ Messmer EM, Aragona $\mathrm{P}$ et al. Br I Ophthalmol 2016:100:300-306.

\section{ABSTRACT}

Meibomian gland dysfunction (MGD) is the most frequent cause of dry eye disease (DED). Eyelid inflammation, microbial growth, associated skin disorders as well as potentially severe corneal complications culminate to make MGD a complex multifactorial disorder. It is probable that MGD is a heterogeneous condition arising from any combination of the following five separate pathophysiological mechanisms: eyelid inflammation, conjunctival inflammation, corneal damage, microbiological changes and DED resulting from tear film instability. The pathogenesis of both MGD and DED can be described in terms of a 'vicious circle': the underlying pathophysiological mechanisms of DED and MGD interact, resulting in a double vicious circle. The MGD vicious circle is self-stimulated by microbiological changes, which results in increased melting temperature of meibum and subsequent meibomian gland blockage, reinforcing the vicious circle of MGD. Meibomian gland blockage, dropout and inflammation directly link the two vicious circles. MGDassociated tear film instability provides an entry point into the vicious circle of DED and leads to hyperosmolarity and inflammation, which are both a cause and consequence of DED. Here we propose a new pathophysiological scheme for MGD in order to better identify the pathological mechanisms involved and to allow more efficient targeting of therapeutics. Through better understanding of this scheme, MGD may gain true disease status rather than being viewed as a mere dysfunction.

\section{INTRODUCTION}

The meibomian glands, found in the upper and lower eyelids, excrete lipids onto the ocular surface that forms the outermost layer of the tear film, lubricating the ocular surface during blinking and protecting against tear evaporation. ${ }^{1}{ }^{2}$ Through dysfunction of the meibomian glands, reduced lipid secretion may contribute to tear film instability and entry into the vicious circle of dry eye disease (DED). ${ }^{3-6}$ Indeed, meibomian gland dysfunction (MGD) is the most common cause of evaporative $\mathrm{DED}^{78}$ and is found even in situations previously considered to be primary (pure) aqueousdeficient DED. ${ }^{9}$ Moreover, MGD is correlated with ocular discomfort during activities requiring relevant visual tasks, such as the use of video display terminals. ${ }^{10}$ Although the precise aetiology and pathophysiology of MGD remain to be determined, in 2011 the International Workshop on MGD proposed the following definition for MGD: "a chronic, diffuse abnormality of the meibomian glands, commonly characterised by terminal duct obstruction and/or qualitative/ quantitative changes in the glandular secretion. It may result in alteration of the tear film, symptoms of eye irritation, clinically apparent inflammation, and ocular surface disease". ${ }^{11}$ The International Workshop on MGD successfully marshalled a large literature base into an exhaustive scheme of the mechanisms underlying the pathogenesis of MGD and the numerous interacting pathways involved. ${ }^{1}$ However, the complexity of this scheme may limit its relevance in clinical practice. Here we introduce a new pathological scheme of MGD, which may be easier to interpret in clinical practice, to facilitate the understanding of the mechanisms that underlie its development and relationship with DED and to allow more efficient treatment of both MGD and DED.

\section{PREVALENCE OF MGD}

Within the general population, precise estimates of MGD prevalence are elusive as rates vary geographically and, until recently, a clear definition of MGD was lacking. The prevalence of MGD varies considerably in published studies. ${ }^{12-16}$ Generally, it is higher in Asian populations, ranging from $46 \%$ to $70 \%$, whereas in Caucasian populations the MGD prevalence ranges from $3.5 \%$ to $20 \%{ }^{11}$ It should be noted that the higher prevalence of MGD in Asian populations is partly due to inconsistent diagnostic criteria among countries. ${ }^{17}$ For example, the Beijing study included both the clinical signs and the symptoms of MGD in their definition whereas other studies did not. ${ }^{7}$ Moreover, certain diagnostic criteria may be unable to distinguish between MGD and aqueous deficient DED, which may also point towards a strong relationship between the two diseases. ${ }^{18}$ The International Workshop on MGD suggests establishing a set of MGD-specific symptoms to aid in diagnosis. ${ }^{7}$ The prevalence of MGD is also affected by age, with older patients at increased risk of developing MGD. In a group of patients aged $<30$ years and $\geq 60$ years, $33 \%$ and $72 \%$ had MGD, respectively. ${ }^{19}$ Prevalence rates also increase if the mixed forms of DED, which include MGD and aqueous tear deficiency, are considered. ${ }^{20}$ 


\section{CLASSIFICATION AND AETIOLOGY OF MGD}

According to the report of the Definition and Classification Subcommittee of the International Workshop on MGD, MGD may be classified as a low- or high-delivery state, according to the extent of meibomian lipid secretion. ${ }^{21}$ The low-delivery state, which is the most common form of MGD, is associated with deficiencies in meibomian secretion, and it may be further characterised as obstructive, with cicatricial and non-cicatricial subcategories, or hyposecretory. ${ }^{21}$ Hyposecretory MGD is associated with gland atrophy. Obstructive MGD is the most prevalent form of low-delivery state MGD and is caused by hyperkeratinisation, which is influenced by sex, hormonal disturbances, topical medications and age. ${ }^{21}$ Interestingly, emerging evidence from both animal and human studies suggests that age-related cell signalling changes within the meibomian gland can lead to gland atrophy, $^{22} 23$ suggesting that some cases of age-related MGD could be classified as hyposecretory rather than obstructive MGD.

Increased meibum viscosity, which is present in all cases of obstructive MGD, may arise because of changes in meibum composition. $^{21}$ High-delivery state MGD, also known as hypersecretory MGD, is characterised by the release of large amounts of meibum at the lid margin in response to pressure on the eyelids. Hypersecretory MGD has been associated with seborrhoeic dermatitis in $100 \%$ of cases, but it may also be associated with rosacea. $^{8} 21$ In both hyposecretory and hypersecretory MGD, the lipids produced are modified (non-canonical), changing the composition and reducing the quality of the tear film, thus leading to symptoms of eye irritation, inflammation and DED. ${ }^{11}$ Although exhaustive, the complexity of the aetiological classification and associated pathological scheme proposed by the International Workshop on MGD may be difficult to interpret in clinical practice as both rare (eg, genetic atrophy of meibomian glands) and more common mechanisms (eg, rosacea) are included at the same level.

Multiple causes may be responsible for the development of MGD-associated tear film alterations, including eye surgery or systemic hormonal treatments such as oestrogen replacement therapy in women and anti-androgen therapy in men. ${ }^{1}$ The latter suggests that hormonal changes contribute to the aetiology of MGD. ${ }^{8}$ Moreover, ophthalmic factors such as aniridia, proliferation of Demodex folliculorum, eyelid tattooing, floppy eyelid syndrome, giant papillary conjunctivitis and trachoma are also believed to correlate with MGD. ${ }^{8}$

\section{IDENTIFYING THE PATHOPHYSIOLOGICAL MECHANISMS OF MGD}

MGD is associated with multiple pathological mechanisms including inflammation, microbial factors and lipid deficiencies. ${ }^{1}$ Eyelid inflammation, microbiological proliferation, release of toxic cytokines onto the cornea and hyper-evaporation combine to create a picture of a benign dysfunction. However, this is often painful and dangerous to the cornea when inflammatory infiltrates, phlyctens, keratitis or peripheral ulcers complicate the meibomitis. ${ }^{8}$ With such variable and complex mechanisms involved in MGD, analysing the pathology and even defining the disease is challenging. Is MGD a disease of the eyelids, tear film, cornea or the entire ocular surface? Moreover, are the associated microbial, metabolic, inflammatory or endocrine diseases related to the eye or the skin? The International Workshop on MGD chose to focus on DED as a consequence of MGD but did not consider meibomitis or eyelid or corneal changes; therefore, the pathophysiology of MGD was not fully resolved. $^{24}$

In our scheme, the stasis of the meibum, which may be caused by obstruction, dropout or inflammation of the meibomian gland, can promote the growth of bacteria. This may then increase the release of esterases and lipases by commensal bacteria of the eyelids. As a consequence of this increased enzyme activity, bacteria can change the viscosity of the meibum, leading to further stasis of the meibum within the meibomian glands, and generate free fatty acids, which in turn causes inflammation and hyperkeratinisation. ${ }^{15}$ These changes in lipid composition may determine the occurrence of foam in the tear film, often visible on the lid margin of patients with MGD. ${ }^{25} 26$

Although a critical analysis of the literature on age-related MGD is beyond the scope of this review, age has been associated with specific pathogenic effects on meibomian gland structure, such as altered localisation of the peroxisome proliferator-activated receptor gamma, a lipid-activated hormone receptor that regulates lipid synthesis and cell differentiation, $^{23} 27$ or meibomian gland atrophy through a loss of MGD progenitors. $^{22}$ Additionally, accumulation of reactive oxygen species with age may play a role in the pathogenesis of MGD. ${ }^{28}$ These mechanisms may underlie atrophic nonobstructive MGD in the older population. ${ }^{29}$ Although the pathophysiology of age-related MGD may be distinct from non-age-related MGD, subsequent meibomian gland dropout ${ }^{29}$ may allow entry into the vicious circle of MGD.

It is likely that MGD is a heterogeneous complex condition arising from any combination of the following five separate pathophysiological mechanisms: eyelid inflammation, conjunctival inflammation, corneal damage, microbiological changes and tear film instability-associated DED. The International Workshop on MGD proposed a complex pathway involved in the self-enforcing vicious circles of MGD (figure 1). ${ }^{21}$ Although these proposed pathways are probably correct, they may be too focused on DED as a final consequence of MGD to be of real practical application. We therefore present the previously published vicious circle of $\mathrm{DED},{ }^{3}$ illustrating its various entry points, and then we build on this to develop the double vicious circle that demonstrates the interacting pathophysiologies of MGD and DED.

\section{VICIOUS CIRCLE OF DED}

In 2007 and 2013 we proposed new patterns for understanding DED (figure 2). ${ }^{3} 6$ Tear film instability, tear hyperosmolarity, apoptosis and inflammation contribute to the pathophysiology of DED. These aetiologies are not mutually exclusive, but rather connected to one another in a cyclical manner, acting as entry points into the vicious circle of DED. As such, DED may be described as an autonomous self-sustaining disease state that is progressively disconnected from its initial causes. In the vicious circle, rapid break-up of the tear film after blinking (tear film instability) leads to local drying and hyperosmolarity of the epithelial surface. In turn, this leads to apoptosis, inflammation and a loss of mucin-producing goblet cells. This cascade of mechanisms, involving osmotic, mechanical and inflammatory stress, destroys goblet cells and defence systems of the ocular surface leading to further damage of the tear film, and thus closes the circle. A major cause such as Sjögren's syndrome can stimulate all states of the vicious circle. Other factors such as corneal surgery, low humidity and high airflow, contact lens wear, allergies or preservatives may disrupt reflex tear delivery to the ocular surface or increase tear film instability, thus initiating entry into the vicious circle. ${ }^{5}$ 


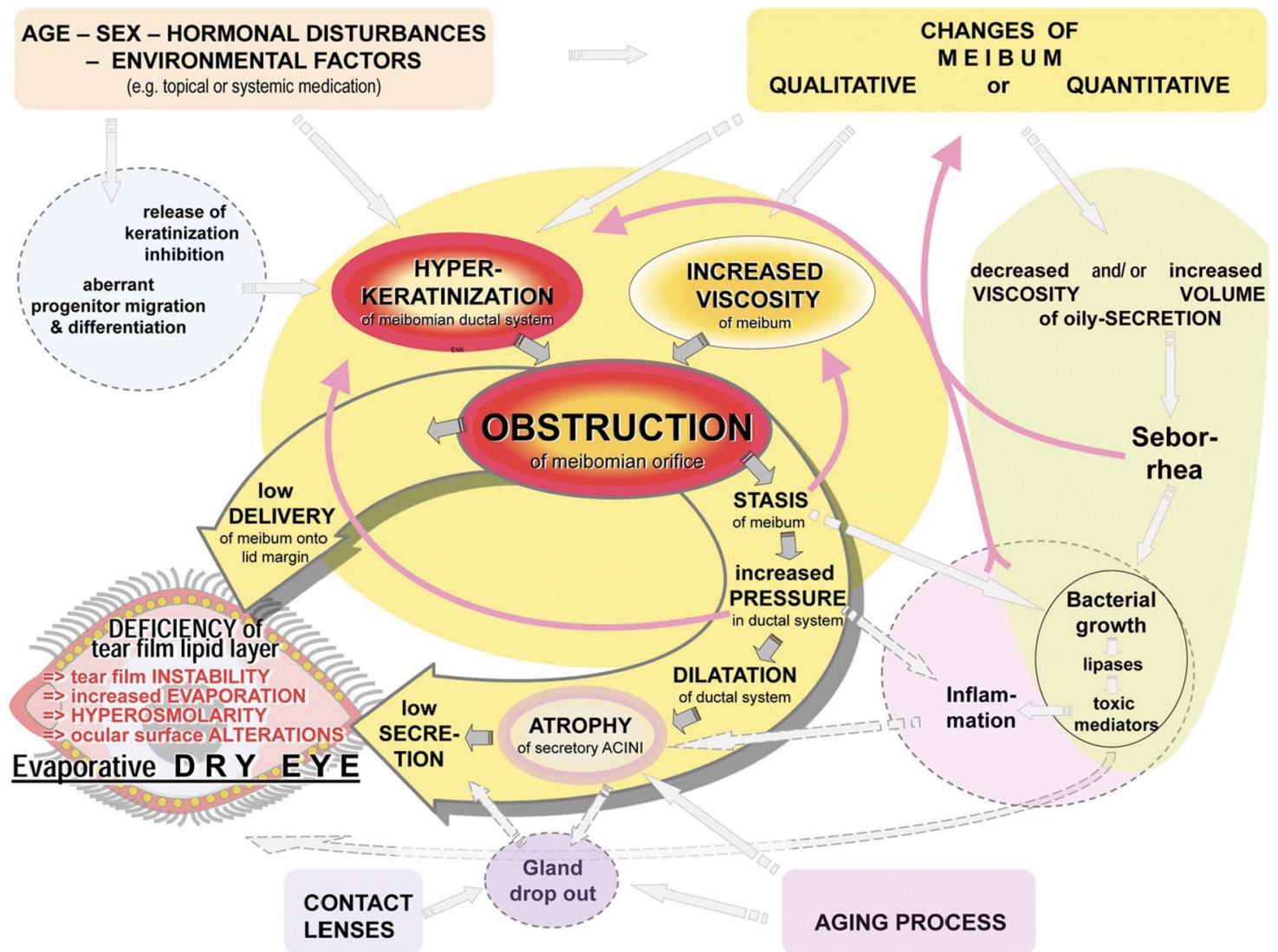

Figure 1 Pathways involved in the pathophysiology of meibomian gland dysfunction (MGD) proposed by the 2011 International Workshop on Meibomian Gland Dysfunction. ${ }^{3} 60$

The vicious circle scheme allows us to understand why, once the cycle is initiated, the continuous environmental challenge acting on a compromised ocular surface allows the vicious circle to perpetuate, even if the initial cause has been removed or reduced (figure 2). ${ }^{3}$ The vicious circle scheme may also promote the development of therapeutic strategies that can simultaneously target the multiple mechanisms underlying the pathophysiology of DED. ${ }^{30}$ For example, tear substitutes with osmoprotective properties ${ }^{31-33}$ may act on multiple points to break the vicious circle of DED. ${ }^{3} 33$ 34 Topical antiinflammatory strategies, such as those containing steroids or cyclosporine, target inflammation and help halt the cycle. ${ }^{35} 36$ Thus, a better understanding of the vicious circle may improve DED management with existing therapies and could also aid in the development of new therapies. We identified MGD as a potential entry point into the vicious circle of DED and, from this, the complexity of the relationship between DED and MGD became apparent. ${ }^{3}$ In order to further elucidate the interacting pathophysiological mechanisms of DED and MGD, we turn to the vicious circle in more detail, this time focusing on MGD.

\section{DED AND MGD: THE DOUBLE VICIOUS CIRCLE}

A new DED scheme that encompassed MGD and illustrated how the related pathophysiological mechanisms underlying DED and MGD combine to form one chronic form of MGD-associated DED needed to be drawn, and this took the form of a double vicious circle illustration (figure 3). Meibomian gland changes act as an entry point into both DED and MGD loops of the double vicious circle; however, as illustrated by the circle, there is no set starting point and MGD can originate elsewhere. Our clinical observations reveal that patients with Sjögren's syndrome can develop MGD through chronic inflammation-induced keratinisation and subsequent dropout and atrophy of the meibomian glands. Although inflammation initiates entry, it is the meibomian gland changes that connect the two vicious circles of MGD and DED. The following four sequential events comprise the MGD loop: stasis of the meibum, bacterial proliferation, release of lipases and esterases, and increased meibum melting temperature. This illustrates how dropout, blockage and/or inflammation of the meibomian glands lead to stasis of the meibum inside the gland, and proliferation of bacteria and mites including Staphylococcus spp and Demodex folliculorum. D. folliculorum is known to promote bacterial proliferation and cause inflammation of the eyelid and possibly the conjunctiva. ${ }^{37} 38$ This ingrowth of bacteria enhances the production of lipid-degrading lipases and esterases that increase the viscosity and melting temperature of the meibum, reducing its secretion onto the surface of the tear film and thus closing the self-sustaining MGD circle. ${ }^{1}$ Furthermore, upper and lower eyelid laxity may exacerbate reduced meibum drainage through decreased muscle pressure on the meibomian glands. ${ }^{35} 39$

Skin diseases (eg, ocular rosacea) are also believed to play a role in MGD pathology; approximately $90 \%$ of patients with ocular rosacea show eyelid changes that are similar to those observed in patients with MGD. ${ }^{40}$ The absence of normal meibum reduces the lipid content of the tear film, allowing entry into the DED loop of the vicious circle, in which the lipiddeficient tear film promotes increased tear evaporation, hyperosmolarity and inflammation. ${ }^{41}$ The missing link between 
Figure 2 Proposed vicious circle of the pathology of dry eye disease. MGD, meibomian gland dysfunction.

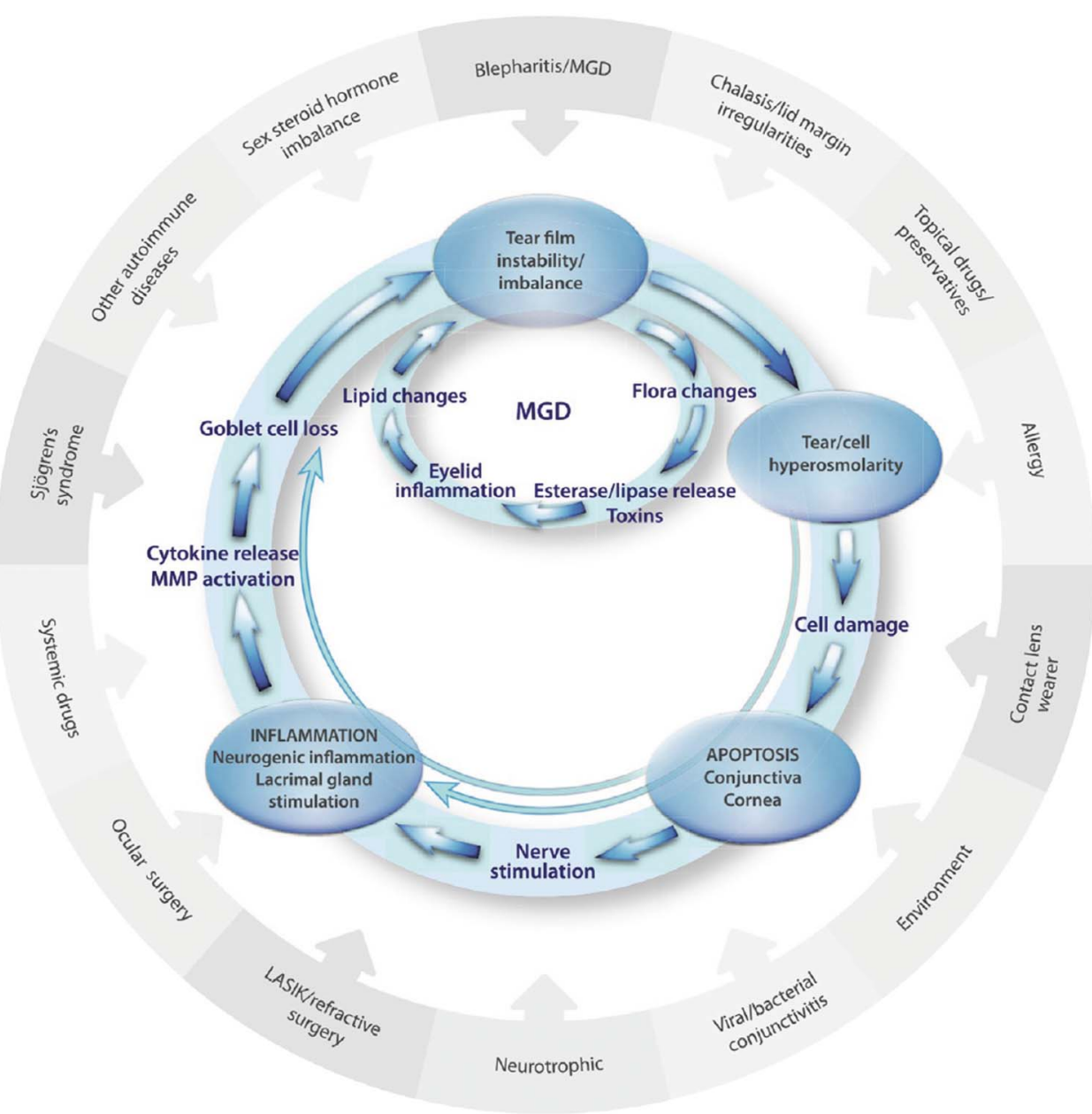

inflammation of the eyelid and lacrimal effects was identified based on the observation that exposure of ocular surface epithelia to desiccating stress led to the release of cornified envelope precursors by the ocular epithelium. ${ }^{42}$ This was accompanied by keratinisation of meibomian gland orifices, which caused further meibomian gland blockade and atrophy as well as loss of mucinfilled goblet cells and entrapment of mucin within the remaining goblet cells, blocking delivery of mucin to the ocular surface and contributing to the development of DED. ${ }^{42}$ Keratinisation of meibomian glands is believed to arise from hyperosmolarity and inflammatory cytokine-induced expression of corneal envelope precursors. ${ }^{43}$ Other pathogenic mechanisms associated with DED include increased age and the use of benzalkonium chloride-containing glaucoma medications. ${ }^{13} 4546$ Meibomian gland blockage, dropout and inflammation directly link the DED and MGD vicious circles.

Although MGD is the most common cause of evaporative DED, ${ }^{78}$ Bron et al ${ }^{41}$ proposed an additional hypothesis where MGD-associated DED leads to a compensatory increase in tear production, followed by compromised lacrimal function and reduced tear secretion that leads to a mixed form of DED comprised of both evaporative and aqueous subtypes. ${ }^{41}$ Support for this hypothesis has been recently published; fluorescein score, tear film break-up time and Schirmer test scores were significantly worse in patients with aqueous-deficient DED compared with MGD, suggesting that increased tear production may compensate, at least for a while, for meibomian gland loss in patients with early-stage MGD. ${ }^{47}$ This hypothesis illustrates how all ocular surface components are inter-related.

Imaging techniques such as in vivo confocal microscopy (IVCM), optical coherence tomography and keratography allow visualisation of the cellular and anatomical structures of the cornea and ocular surface. ${ }^{48-50}$ These techniques can therefore provide valuable information on the relationship between DED and MGD. ${ }^{51}$ Infrared imaging revealed an increased meibomian gland dropout score in patients with Sjögren's syndrome compared with control subjects without DED, ${ }^{49}$ suggesting that patients with Sjögren's syndrome may be at a greater risk of developing MGD. Moreover, IVCM revealed that the meibomian glands of patients with Sjögren's syndrome had higher acinar density, smaller diameters, a greater density of periglandular inflammatory cells and higher secretion reflectivity compared with patients with MGD. ${ }^{51}$ These observations support an essential role of MGD in DED and could provide a histopathological basis for the previously mentioned mixed form of aqueous and evaporative DED. Additionally, in a retrospective observational pilot study of patients with MGD-associated refractory DED, IVCM revealed clinically non-apparent inflammation of the palpebral conjunctiva despite improvements in tear film break-up time, an increased number of meibomian glands yielding secretion and a normal clinical examination post-treatment, suggesting that IVCM may also be a useful tool for identifying the underlying causes of symptom-sign disparity in patients with MGD. ${ }^{52}$ Further studies are needed to truly 


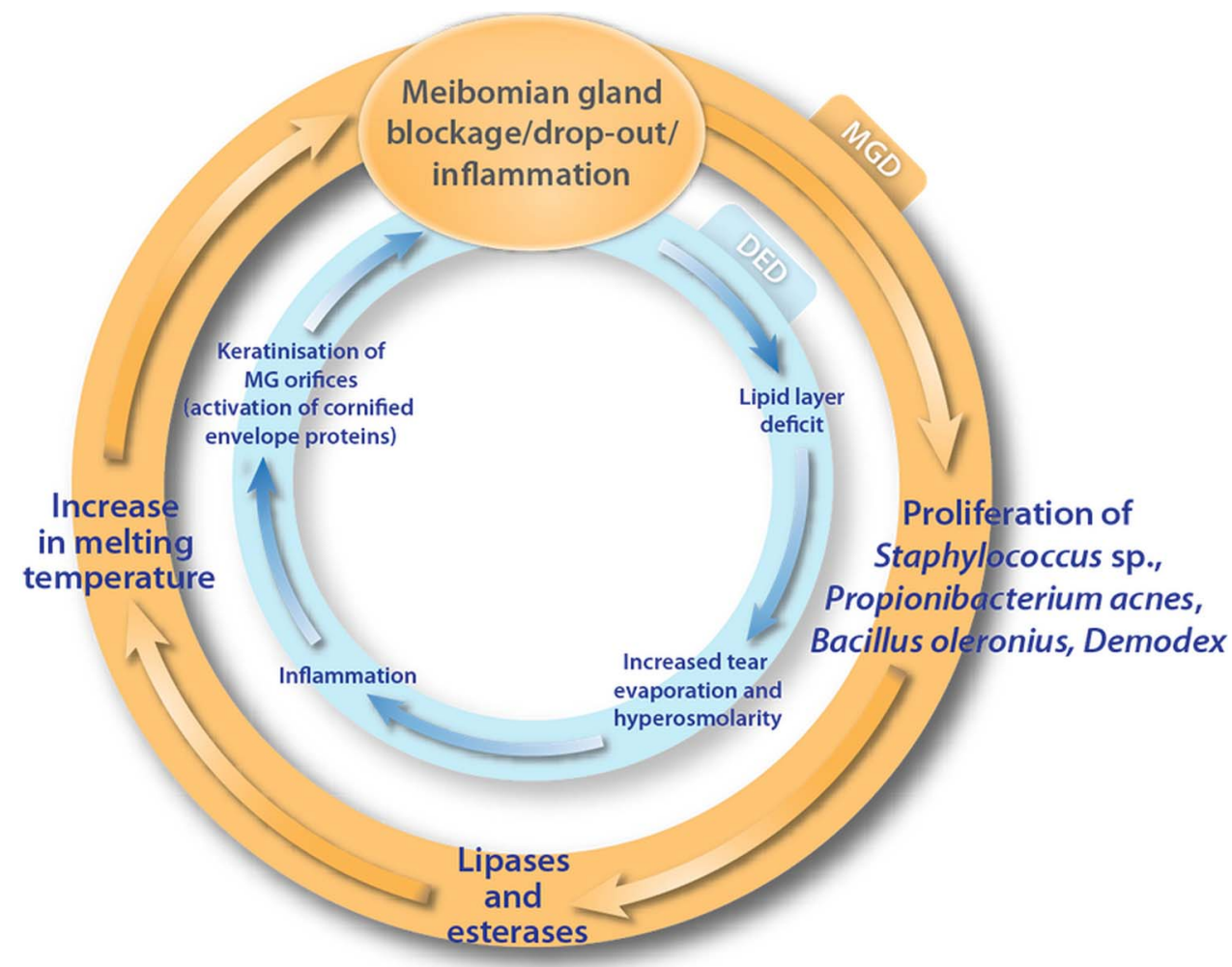

Figure 3 Importance of meibomian gland dysfunction in the vicious circle of the pathology of dry eye disease. ${ }^{61}$

understand how imaging can advance our understanding of the relationship between MGD and DED.

\section{WHAT IS THE PURPOSE OF THIS NEW DOUBLE VICIOUS \\ CIRCLE?}

Beyond its educational and scientific interest, the double vicious circle may be used for understanding and revealing the complexity of the pathophysiological mechanisms underlying DED and MGD, as well as a tool to focus and guide therapy. Figure 4 illustrates how different therapies, some acting at multiple points of the circle, may be used to disrupt the vicious circles of MGD and DED. Eyelid hygiene, consisting of eyelid warming and massage, reduces the proliferation of bacteria that are believed to increase the melting temperature of meibum, in

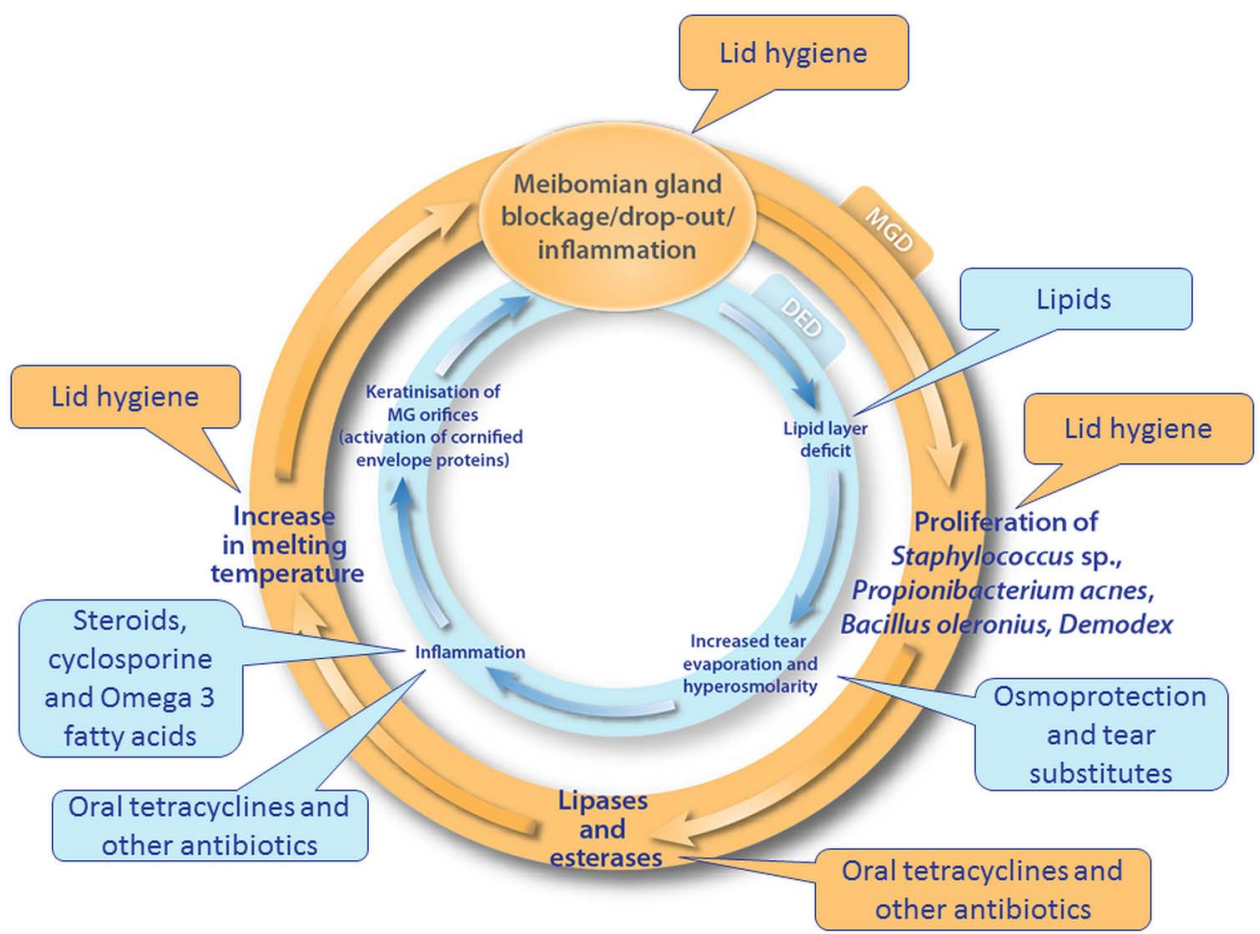

Figure 4 Different therapies may target particular pathophysiological mechanisms that underlie the vicious circle of dry eye disease. 
addition to melting the altered meibomian lipids to improve their secretion. ${ }^{35}{ }^{53}$ Eyelid hygiene devices such as the MGDRx Eyebag (The Eyebag Company, West Yorkshire, UK), Blephasteam (Laboratoires Théa, Clermont-Ferrand, France) and LipiFlow (TearScience, North Carolina, USA) have been shown to improve symptoms in patients with MGD. ${ }^{54-57}$ The anti-inflammatory and antimicrobial effects of oral tetracycline derivatives such as doxycycline, and antibiotics, including azithromycin, may reduce bacterial proliferation and prevent inflammation-induced keratinisation of meibomian glands to improve meibum secretion. ${ }^{35} 5859$ Moreover, figure 4 illustrates how therapies that have the potential to break one of the vicious circles, such as tear substitutes, may impact on the other via indirect effects on meibomian gland blockage, dropout and inflammation.

It seems apparent that a diagnosis of MGD is based on a set of signs and symptoms and that its true nosology remains to be determined. The double vicious circle scheme defined here creates the foundation for the classification of MGD; it highlights the pathophysiological mechanisms involved and addresses the basics of treatment. Taken together, this new scheme perhaps gives MGD true disease status and identifies it as more than a simple dysfunction.

\footnotetext{
Author affiliations

${ }^{1}$ Quinze-Vingts National Ophthalmology Hospital, University Versailles St Quentin en Yvelines, Versailles, France

2UPMC Univ Paris 06, UMR S 968, Institut de la Vision, Paris, France

${ }^{3}$ Department of Ophthalmology, Ludwig Maximilian University, Munich, Germany

${ }^{4}$ Institute of Ophthalmology, University of Messina, Messina, Italy

${ }^{5}$ Department of Ophthalmology, University Hospital Düsseldorf, Heinrich-Heine University, Düsseldorf, Germany

${ }^{6}$ Department of Ophthalmology, Bayindir Hospital, Ankara, Turkey

${ }^{7}$ San Carlos University Hospital, Complutense University, Madrid, Spain

${ }^{8}$ Department of Ophthalmology, Aristotle University of Thessaloniki, Thessaloniki, Greece

${ }^{9}$ University of Oviedo and Fernández-Vega Ophthalmological Institute, Oviedo, Spain

${ }^{10}$ Department of Neuroscience, Ophthalmology, and Genetics, University of Genoa, Genoa, Italy

${ }^{11}$ Ophthalmology Department, Bicêtre University Hospital, Le Kremlin-Bicêtre, France
}

\section{Twitter Follow Kostas Boboridis at @KBoboridis}

Acknowledgements The authors thank Newton Healthcare Communications for writing and editorial support, which was funded by Allergan, UK.

Contributors $C B$ defined the concept and scope of the review. EMM, PA, GG, YAA, JBdC, KGB, JM-L, MR and ML provided critical review of the manuscript. All of the authors were involved in the finalisation of the manuscript.

Funding Allergan provided funding for the meetings and for the development of this manuscript. The authors were involved with the entire process, from design to critical revision of the manuscript, and maintained complete control over the direction and content of the paper.

Competing interests $C B$ has received research grants and consulting fees from Alcon, Allergan, Santen and Théa. EMM has acted as an advisor and presenter for Allergan, Alcon, Bausch \& Lomb, Croma-Pharma, MSD, Oculus, Santen, Théa and Ursapharm. PA has acted as a consultant and presenter for Allergan, Alcon Italy, Bausch \& Lomb, Santen, Medivis, Théa, Eupharmed and Farmigea and has received a research grant from SOOFT Italia. GG has acted as a consultant or speaker for Allergan, Alcon, Bausch \& Lomb, Chiesi, Oculus, Santen, Théa, TearLab and TearScience. YAA has acted as an advisor and presenter for Allergan, Théa, Bausch \& Lomb, and Alcon. JBdC has acted as a consultant for Allergan, Bausch \& Lomb, Théa, Alcon and Santen. KGB declares financial relationships with Allergan, Alcon and Théa. MR declares financial relationships with Allergan, Bausch \& Lomb, Farmigea, Théa, Alcon, Eupharma, Santen/Novagali and Alfalntes. JM-L has received research grants from Théa and has acted as a consultant for Allergan. ML has acted as a consultant for Allergan, Alcon, Bausch \& Lomb, Farmigea, MSD, Santen/ Novagali and Théa.

Provenance and peer review Not commissioned; externally peer reviewed.

Open Access This is an Open Access article distributed in accordance with the Creative Commons Attribution Non Commercial (CC BY-NC 4.0) license, which permits others to distribute, remix, adapt, build upon this work non-commercially, and license their derivative works on different terms, provided the original work is properly cited and the use is non-commercial. See: http://creativecommons.org/ licenses/by-nc/4.0/

\section{REFERENCES}

1 Knop E, Knop N, Millar T, et al. The International Workshop on Meibomian Gland Dysfunction: report of the Subcommittee on Anatomy, Physiology, and Pathophysiology of the Meibomian Gland. Invest Ophthalmol Vis Sci 2011;52:1938-78.

2 McCulley JP, Shine WE. Meibomian gland function and the tear lipid layer. Ocul Surf 2003;1:97-106.

3 Baudouin C, Aragona P, Messmer EM, et al. Role of hyperosmolarity in the pathogenesis and management of dry eye disease: proceedings of the OCEAN group meeting. Ocul Surf 2013;11:246-58.

4 Rolando M, Papadia M. Diagnosis and management of the lid and ocular surface disorders. In: Asbell PA, Lemp MA, eds. Dry eye disease: the clinician's guide to diagnosis and treatment. New York: Thieme Medical Publishers, 2006:63-83.

5 International Dry Eye Workshop. Report of the International Dry Eye Workshop (DEWS). Ocul Surf 2007;5:61-204.

6 Baudouin C. [A new approach for better comprehension of diseases of the ocular surface]. J Fr Ophtalmol 2007;30:239-46.

7 Schaumberg DA, Nichols JJ, Papas EB, et al. The International Workshop on Meibomian Gland Dysfunction: report of the subcommitee on the epidemiology of, and associated risk factors for, MGD. Invest Ophthalmol Vis Sci 2011:52:1994-2005.

8 Foulks GN, Nichols KK, Bron AJ, et al. Improving awareness, identification, and management of meibomian gland dysfunction. Ophthalmology 2012;119:S1-12.

9 Lemp MA, Foulks GN, Pepose JS. Evaluation of tear osmolarity in non-Sjogren and Sjogren syndrome dry eye patients with the TearLab system. Cornea 2013;32:379-81.

10 Fenga C, Aragona P, Cacciola A, et al. Meibomian gland dysfunction and ocular discomfort in video display terminal workers. Eye 2008;22:91-5.

11 Nichols KK, Foulks GN, Bron AJ, et al. The International Workshop on Meibomian Gland Dysfunction: Executive Summary. Invest Ophthalmol Vis Sci 2011:52:1922-9.

12 Uchino M, Dogru M, Yagi Y, et al. The features of dry eye disease in a Japanese elderly population. Optom Vis Sci 2006:83:797-802.

13 Schein OD, Munoz B, Tielsch JM, et al. Prevalence of dry eye among the elderly. Am J Ophthalmol 1997:124:723-8.

14 McCarty CA, Bansal AK, Livingston PM, et al. The epidemiology of dry eye in Melbourne, Australia. Ophthalmology 1998;105:1114-19.

15 Lekhanont K, Rojanaporn D, Chuck RS, et al. Prevalence of dry eye in Bangkok, Thailand. Cornea 2006:25:1162-7.

16 Lin PY, Tsai SY, Cheng CY, et al. Prevalence of dry eye among an elderly Chinese population in Taiwan: the Shihpai Eye Study. Ophthalmology 2003;110:1096-101.

17 Viso E, Rodriguez-Ares MT, Abelenda D, et al. Prevalence of asymptomatic and symptomatic meibomian gland dysfunction in the general population of Spain. Invest Ophthalmol Vis Sci 2012;53:2601-6.

18 Arita R, Itoh K, Maeda S, et al. Efficacy of diagnostic criteria for the differential diagnosis between obstructive meibomian gland dysfunction and aqueous deficiency dry eye. Jpn J Ophthalmol 2010;54:387-91.

19 Stanek S. Meibomian gland status comparison between active duty personnel and U.S. veterans. Mil Med 2000;165:591-3.

20 Tong L, Chaurasia SS, Mehta JS, et al. Screening for meibomian gland disease: its relation to dry eye subtypes and symptoms in a tertiary referral clinic in Singapore. Invest Ophthalmol Vis Sci 2010;51:3449-54

21 Nelson JD, Shimazaki J, Benitez-del-Castillo JM, et al. The International Workshop on Meibomian Gland Dysfunction: report of the Definition and Classification Subcommittee. Invest Ophthalmol Vis Sci 2011;52:1930-7.

22 Parfitt GJ, Xie Y, Geyfman M, et al. Absence of ductal hyper-keratinization in mouse age-related meibomian gland dysfunction (ARMGD). Aging (Albany NY) 2013;5:825.

23 Nien CJ, Massei S, Lin G, et al. Effects of age and dysfunction on human meibomian glands. Arch Ophthalmol 2011;129:462-9.

24 Foulks GN, Bron AJ. Meibomian gland dysfunction: a clinical scheme for description, diagnosis, classification, and grading. Ocul Surf 2003;1:107-26.

25 Lozato PA, Pisella PJ, Baudouin C. [The lipid layer of the lacrimal tear film: physiology and pathology]. J Fr Ophtalmol 2001;24:643-58.

26 Borchman D, Foulks GN, Yappert MC, et al. Human meibum lipid conformation and thermodynamic changes with meibomian-gland dysfunction. Invest Ophthalmol Vis Sci 2011;52:3805.

27 Nien CJ, Paugh JR, Massei $\mathrm{S}$, et al. Age-related changes in the meibomian gland. Exp Eye Res 2009;89:1021-7

28 Ibrahim OM, Dogru M, Matsumoto $Y$, et al. Oxidative stress induced age dependent meibomian gland dysfunction in Cu, Zn-superoxide dismutase-1 (Sod1) knockout mice. PLOS ONE 2014;9:e99328. 
29 Villani E, Canton V, Magnani F, et al. The aging meibomian gland: an in vivo confocal study. Invest Ophthalmol Vis Sci 2013;54:4735-40.

30 Labetoulle M, Baudouin C. From pathogenic considerations to a simplified decision-making schema in dry eye disease. J Fr Ophtalmol 2013;36:543-7.

31 Hamano $T$, Horimoto $K$, Lee $M$, et al. Sodium hyaluronate eyedrops enhance tear film stability. Jpn J Ophthalmol 1996;40:62-5.

32 Nakamura M, Hikida M, Nakano T, et al. Characterization of water retentive properties of hyaluronan. Cornea 1993:12:433-6.

33 Simmons PA, Chang-Lin J-E, Chung Q, et al. Effect of compatible solutes on transepithelial electrical resistance and uptake in primary rabbit corneal epithelial cell layers model. Presented at the Association for Research in Vision and Ophthalmology (ARVO), 2007.

34 Bayhan SA, Bayhan HA, Muhafiz E, et al. Effects of osmoprotective eye drops on tear osmolarity in contact lens wearers. Can J Ophthalmol 2015;50:283-9.

35 Geerling G, Tauber J, Baudouin C, et al. The International Workshop on Meibomian Gland Dysfunction: report of the Subcommittee on Management and Treatment of Meibomian Gland Dysfunction. Invest Ophthalmol Vis Sci 2011;52:2050-64.

36 Qiao J, Yan X. Emerging treatment options for meibomian gland dysfunction. Clin Ophthalmol 2013;7:1797-803.

37 Kosik-Bogacka DI, Lanocha N, Lanocha A, et al. Role of Demodex folliculorum in the pathogenesis of blepharitis. Acta Ophthalmol 2012;90:e579.

38 Murillo N, Aubert J, Raoult D. Microbiota of Demodex mites from rosacea patients and controls. Microb Pathog 2014;71-72:37-40

39 Chang M, Lee $\mathrm{H}$, Park MS, et al. The clinical characteristics and new classification of sticky eyelid syndrome in East Asian patients. Acta Ophthalmol 2014;92: e667-70.

40 Alvarenga LS, Mannis MJ. Ocular rosacea. Ocul Surf 2005;3:41-58.

41 Bron AJ, Yokoi N, Gafney E, et al. Predicted phenotypes of dry eye: proposed consequences of its natural history. Ocul Surf 2009;7:78-92.

42 Corrales RM, de Paiva CS, Li DQ, et al. Entrapment of conjunctival goblet cells by desiccation-induced cornification. Invest Ophthalmol Vis Sci 2011;52:3492-9.

43 Takahashi H, Asano-Kato N, Nakamura S, et al. Interferon-gamma-dependent stimulation of human involucrin gene expression: STAT1 (signal transduction and activators of transcription 1) protein activates involucrin promoter activity. Biochem J 1999;334:797-802.

44 Chen Z, Tong L, Li Z, et al. Hyperosmolarity-induced cornification of human corneal epithelial cells is regulated by JNK MAPK. Invest Ophthalmol Vis Sci 2008:49:539-49.

45 Schein OD, Hochberg MC, Munoz B, et al. Dry eye and dry mouth in the elderly: a population-based assessment. Arch Intern Med 1999;159:1359-63.

46 Pisella PJ, Pouliquen P, Baudouin C. Prevalence of ocular symptoms and signs with preserved and preservative free glaucoma medication. $\mathrm{Br} J$ Ophthalmol 2002;86:418-23.
47 Arita R, Morishige N, Koh S, et al. Increased tear fluid production as a compensatory response to meibomian gland loss: a multicenter cross-sectional study. Ophthalmology 2015;122:925-33.

48 Qazi Y, Aggarwal S, Hamrah P. Image-guided evaluation and monitoring of treatment response in patients with dry eye disease. Graefes Arch Clin Exp Ophthalmol 2014;252:857-72.

49 Menzies KL, Srinivasan S, Prokopich CL, et al. Infrared imaging of meibomian glands and evaluation of the lipid layer in Sjögren's syndrome patients and nondry eye controls. Invest Ophthalmol Vis Sci 2015;56:836-41.

50 Ban Y, Ogawa Y, Ibrahim OM, et al. Morphologic evaluation of meibomian glands in chronic graft-versus-host disease using in vivo laser confocal microscopy. Mol Vis 2011:17:2533.

51 Villani E, Beretta S, De CM, et al. In vivo confocal microscopy of meibomian glands in Sjogren's syndrome. Invest Ophthalmol Vis Sci 2011;52:933-9.

52 Qazi Y, Kheirkhah A, Blackie C, et al. In vivo detection of clinically non-apparent ocular surface inflammation in patients with meibomian gland dysfunction-associated refractory dry eye symptoms: a pilot study. Eye 2015;29:1099-110

53 Gao YY, Di Pascuale MA, Li W, et al. In vitro and in vivo killing of ocular Demodex by tea tree oil. Br J Ophthalmol 2005;89:1468-73.

54 Bilkhu PS, Naroo SA, Wolffsohn JS. Randomised masked clinical trial of the MGDRx eyebag for the treatment of meibomian gland dysfunction-related evaporative dry eye. Br J Ophthalmol 2014;98:1707-11.

55 Benitez Del Castillo JM, Kaercher T, Mansour K, et al. Evaluation of the efficacy, safety, and acceptability of an eyelid warming device for the treatment of meibomian gland dysfunction. Clin Ophthalmol 2014;8:2019-27.

56 Finis D, Hayajneh J, Konig C, et al. Evaluation of an automated thermodynamic treatment (LipiFlow): system for meibomian gland dysfunction: a prospective, randomized, observer-masked trial. Ocul Surf 2014;12:146-54.

57 Doan S, Chiambaretta F, Baudouin C. Evaluation of an eyelid warming device (Blephasteam) for the management of ocular surface diseases in France: the ESPOIR study. J Fr Ophtalmol 2014;37:763-72.

58 Aghai ZH, Kode A, Saslow JG, et al. Azithromycin suppresses activation of nuclear factor-kappa $B$ and synthesis of pro-inflammatory cytokines in tracheal aspirate cells from premature infants. Pediatr Res 2007;62:483-8.

59 Li DQ, Chen Z, Song XJ, et al. Stimulation of matrix metalloproteinases by hyperosmolarity via a JNK pathway in human corneal epithelial cells. Invest Ophthalmol Vis Sci 2004;45:4302-11.

60 Knop E, Knop N. [Meibomian glands : part IV. Functional interactions in the pathogenesis of meibomian gland dysfunction (MGD)]. Ophthalmologe 2009;106:980-7.

61 Baudouin C. [Revisiting meibomian gland dysfunction]. J Fr Ophtalmol 2014;37:757-62. 